\title{
Kontexte des Wohlbefindens von Kindern und Jugendlichen. Integrierende Stadtentwicklung mit dem kommunalen kleinräumigen Monitoring-Instrument UWE
}

\section{Contexts of children's well-being. Integrating urban development by the municipal small- scale monitoring instrument UWE}

https://doi.org/10.2478/rara-2019-0034

Eingegangen: 11. Juni 2018 / Angenommen: 25. April 2019

Zusammenfassung: Dieser Beitrag stellt Ergebnisse einer Pilotstudie vor, die ein Monitoring-Instrument des Wohlbefindens von Kindern und Jugendlichen für die kommunale Dauerbeobachtung und als Evaluationsinstrument entwickelte. Integrierende Stadtentwicklung steht vor der Herausforderung, negative Folgen von residentieller Segregation und Bildungssegregation für die Entwicklung von Kindern und Jugendlichen abzufedern. Zugleich mangelt es Städten an systematisch verfügbaren kleinräumigen Daten über Kinder und Jugendliche im Sekundarstufenalter. Das Instrument setzt am Humanvermögen als gesellschaftsintegrativem Potenzial an und fragt, wie Wohlbefinden von spezifischen Ressourcen und sozialräumlichen Kontexten beeinflusst wird, in denen sich für die Entwicklung prägende Interaktionen abspielen: Familie, Schule, Wohnumgebung. Die gleichzeitige Untersuchung verschiedener sozialräumlicher Kontexte erlaubt differenzierte Interventionsmöglichkeiten, die ressourcenspezifisch eingesetzt werden können.

Abstract: This article presents results of a pilot-study, which developed a monitoring-instrument for measuring wellbeing of children and young people in a municipal permanent observation and as evaluation tool. Integrated urban development faces the challenge to absorb negative consequences at the development of children by residential and educational segregation. At the same time, in cities there is a lack of systematic available small-scale data about children in the middle years. The instrument uses human resources as necessary potential for society and measures areas of children's development strongly linked to well-being and the influence by the socio-spatial contexts: family, school and living environment. A simultaneous testing of these contexts allows options of intervention, which can be used resource-specific.

Schlüsselwörter: Wohlbefinden von Kindern, sozialräumlicher Kontext, Familie, Schule, Wohnumgebung, Monitoring-Instrument

Keywords: Children's well-being, Socio-spatial context, Family, School, Living environment, Monitoring instrument

\footnotetext{
*Corresponding author: Prof. Dr. Sören Petermann, Zentrum für interdisziplinäre Regionalforschung an der Ruhr-Universität Bochum Im Lottental 38, 44801 Bochum, Deutschland, E-mail: soeren.petermann@rub.de

Prof. Dr. Klaus Peter Strohmeier, Katharina Yaltzis: Zentrum für interdisziplinäre Regionalforschung an der Ruhr-Universität Bochum Im Lottental 38, 44801 Bochum, Deutschland
} 


\section{Einleitung}

Dieser Beitrag stellt Ergebnisse einer Untersuchung zum Einfluss sozialräumlicher Kontexte auf das Wohlbefinden von Kindern und Jugendlichen im Sekundarstufenalter vor. ${ }^{1}$ Die Untersuchung ist eine wichtige Etappe zur Entwicklung kommunaler kleinräumiger MonitoringInstrumente. Wir fokussieren uns auf sozialräumliche Kontexte, in denen Kinder und Jugendliche heranwachsen und wichtige kognitive und soziale Fähigkeiten und Kompetenzen entwickeln. Diese Kontexte sind in kommunaler Sozialpolitik zu berücksichtigen und somit in der Stadtentwicklung zu verankern.

Der von uns verfolgte Ansatz leistet in zweierlei Hinsicht wichtige Beiträge für eine integrierende Stadtentwicklung. Zum einen setzt er an der sozialen innerstädtischen Spaltung in Form residentieller Segregation und Bildungssegregation an und fragt nach den Auswirkungen dieser Spaltungen für das Aufwachsen der Kinder und Jugendlichen. Zum anderen berichten wir nicht nur über, sondern vor allem mit Kindern und Jugendlichen und zwar in und aus einer Lebensphase, über die kommunalen Sozialplanern in der Regel kaum umfangreiche systematische kleinräumige Statistiken und Erkenntnisse vorliegen.

Eine zentrale Herausforderung integrierender Stadtentwicklung ist die Abfederung negativer Folgen von residentieller Segregation und Bildungssegregation für die Entwicklung von Kindern und Jugendlichen. Die Bildungssegregation an Schulen entsteht teilweise aus der institutionellen Trennung von Kindern und Jugendlichen in Hauptschulen, Realschulen, Gesamtschulen, Gymnasien und integrierten Schulformen (Wößmann 2009). Welche Schule Kinder und Jugendliche besuchen, beispielsweise ein Gymnasium, wird von ihrer sozialen Herkunft beeinflusst. Sozial begünstigte Kinder und Jugendliche haben hierzulande eine viel größere Chance auf einen Gymnasialbesuch als solche aus Arbeiterfamilien (Stubbe/Bos/Euen 2012). Aber auch der Wohnort und das Aufwachsen in bestimmten Stadtteilen beeinflussen die schulische Laufbahn stark. Übergänge auf weiterführende Schulen folgen dem Muster sozialer und sozialräumlicher Ungleichheiten (vgl. Terpoorten 2014).

Es gibt zwar Studien über die Erfahrung von Armut (vgl. Frankfurter Kinderbefragung; Hallmann/Beisenkamp/Klöckner et al. 2008), es fehlt aber an spezifischen

1 Die Benennung der Zielgruppe (Kinder und Jugendliche im Sekundarstufenalter) ist sehr sperrig. Für einen besseren Lesefluss verkürzen wir oft zu „Kinder und Jugendliche“, gelegentlich nur zu „Kinder“ oder nur zu „Jugendliche“.
Messungen, wie Kinder und Jugendliche das Aufwachsen in konkreten sozial segregierten Wohnumgebungen und in konkreten segregierten Lernorten (Schulen) in einer Stadt erleben. Erste Forschungen zur subjektiven Sicht von Kindern auf ihr eigenes benachteiligtes Quartier zeigen, dass es für die Stadtforschung und für die praktische Stadtentwicklung sinnvoll sein kann, Kinder als Experten zu betrachten und ernst zu nehmen. In diesem Expertenstatus sollten sie eine aktive Rolle in der Quartiersentwicklung spielen (vgl. Hilke 2013; Kothe 2014).

Es geht bei unserem Ansatz also um zweierlei: die Erhebung von Faktoren, die die Entwicklung von Kindern und Jugendlichen in der Stadt beeinflussen, um mit diesem Wissen persönliche Entwicklungsprozesse möglichst erfolgreich gestalten zu können, und die Erhebung der subjektiven Sicht von kindlichen und jugendlichen Experten auf ihre Wohn- und Lernorte in der Stadt, um diese verbessern zu können.

Das LBS-Kinderbarometer (Beisenkamp/Klöckner/ Hallmann et al. 2009) zeigt (allgemein!), dass das Wohlbefinden von Kindern im Alter von neun bis 14 Jahren stark von informellen Lebensbereichen, wie Familie und Freundeskreis, abhängt. Danach kommt die Schule, aber hier geht es eher nicht um ihre Qualität als Ort formalen Lernens. Diese allgemeinen Informationen helfen der kommunalen Politik und der Stadtgesellschaft nicht. Wer Kinder effektiv fördern will, muss die für sie relevanten Probleme und Ressourcen kennen. Voraussetzung für die effektive Förderung benachteiligter Kinder und die Entwicklung ihrer Kontexte sind kleinräumige und institutionenscharfe Indikatoren, mit denen Akteure vor Ort informiert werden, wo Förderung und Veränderung besonders gebraucht werden (Diagnose) und welche Wirkungen gegebenenfalls erreicht worden sind (Evaluation). Vorhandene einschlägige Studien (z. B. Hallmann/ Beisenkamp/Klöckner et al. 2008) sind zwar repräsentativ, erlauben aber nur unspezifische Diagnosen, denn sie sind nicht schul- oder sozialraumspezifisch auswertbar, was ihren praktischen Nutzen einschränkt. Die amtliche Statistik in Nordrhein-Westfalen kann zwar auf der Ebene der Städte und Gemeinden Daten bereitstellen (vgl. die Onlineangebote "Wegweiser Kommune“ der Bertelsmann-Stiftung ${ }^{2}$ ), sie ist jedoch nicht geeignet für Vergleiche von Sozialräumen oder von Unterschieden zwischen Kindern verschiedener Schulen oder Tageseinrichtungen. Die wenigsten Kommunen in NordrheinWestfalen können (oder wollen) heute irgendwelche kleinräumigen oder gar institutionenscharfen Daten

2 http://www.wegweiser-kommune.de/ (04.03.2019). 
liefern. Über die Lebensqualität, die Lebenschancen, die Gesundheit oder das Befinden von Kindern erfahren wir auf diese Weise von den Kommunen nichts.

Eine konkrete praktische Herausforderung integrierender Stadtentwicklung ist somit der Aufbau eines Monitoring-Systems für Kommunen zum Wohlbefinden von Kindern und Jugendlichen in der Sekundarstufe für die Dauerbeobachtung und als Evaluationsinstrument. Ein solches Monitoring-System kann praktische Ansatzpunkte für eine integrierende Stadtentwicklung identifizieren, die gleichwertige Lebensverhältnisse der Familien und gleichwertige Entwicklungschancen für die Kinder in den Sozialräumen einer Stadt verwirklichen will. Es ist ein populationsdiagnostisches Instrument, das kleinräumig und institutionenscharf statistische Informationen bereitstellt und von Einzelfällen abstrahiert. Damit ist gemeint, dass wir ein Monitoring-Instrument vorstellen, das über die ,flächendeckende' standardisierte und damit vergleichbare Befragung von Kindern und Jugendlichen Aussagen zum Wohlbefinden trifft.

Die Forschungsfrage dieses Beitrags lautet: Wie lässt sich der Einfluss sozialräumlicher Kontexte auf das Wohlbefinden von Kindern und Jugendlichen im Rahmen eines kommunalen Monitoring-Instruments diagnostizieren und evaluieren? Der Beitrag wird im Folgenden zunächst knapp die Pilotstudie in der Stadt Herne vorstellen, die die Entwicklung eines erprobten MonitoringInstruments zum Ziel hat (Kapitel 2). Im dritten Kapitel werden der theoretische Rahmen für die Analyse der Kontextabhängigkeit des kindlichen Wohlbefindens erläutert und daraus abgeleitete Hypothesen vorgestellt. Das vierte Kapitel beschreibt das methodische Vorgehen und die Analysestrategie. Die in mehreren Schritten verlaufende Auswertung der Daten ist Gegenstand des fünften Kapitels. Im letzten Kapitel werden die Ergebnisse im Hinblick auf die Hypothesen zusammengefasst und kritisch eingeordnet. Der Beitrag endet mit einem Ausblick, der knapp den Transfer in die Praxis umreißt und weiterführende Fragen aufwirft.

\section{Die Pilotstudie}

Kern der Studie ist das Monitoring-Instrument UWE. UWE steht für „Umwelt, Wohlbefinden und Entwicklung von Kindern und Jugendlichen in Kommunen“. Kern des Instruments ist die Befragung „Wie geht's dir? UWE“, die auf Selbsteinschätzungen basiert. Es wird untersucht, ob und unter welchen Umständen sich Kinder glücklich fühlen, wie gesund sie sich fühlen, wie es um ihr Selbstvertrauen, ihre Ängste, ihren Optimismus steht und von welchen Bedingungen und Ressourcen ihr Wohlbefinden abhängt. UWE gibt Kindern und Jugendlichen eine Stimme und damit eine Möglichkeit, den Erwachsenen in ihren Kontexten mitzuteilen, wie ihre Erfahrungen innerhalb und außerhalb der Schule sind. Ebenso liefern die Ergebnisse Wissenschaftlern und politischen Entscheidungsträgern wichtige Informationen und einen Einblick in die Lebenserfahrungen von Kindern. Diese Ergebnisse ermöglichen, die sozialräumlichen Kontexte, in denen Kinder ihren Alltag verbringen, möglichst optimal zu gestalten.

Die Studie ist eine Adaption des kanadischen „Middle Years Development Instrument" (MDI). ${ }^{3}$ Das MDI ist ein Selbstbeurteilungsfragebogen, den Schüler der vierten und siebten Klassen in Kanada beantworten. In der deutschen UWE-Studie werden Kinder und Jugendliche der siebten und neunten Klassen weiterführender Schulen über ihre Gedanken, Gefühle und Erfahrungen in den Kontexten Familie, Schule und Wohnumgebung befragt. UWE ist ein in Deutschland bislang einzigartiges umfassendes Monitoring-Instrument, das dabei hilft, ein tieferes Verständnis dafür zu bekommen, wie Kinder und Jugendliche in dieser Lebensphase ihre sozialen und räumlichen Kontexte erleben. Für die Praxis ergeben sich Orientierungen und praktische Ansätze für Veränderungen, denn durch UWE entstehen Gesprächsanlässe mit lokalen Partnern vor allem aus dem Sozial- und Bildungsbereich und aus anderen Bereichen. Daraus lassen sich für die Kommune wichtige Impulse ableiten, um gemeinsame Handlungsstrategien in lokalen Netzwerken voranzubringen.

Die Umfrage sammelt Informationen über schulische und außerschulische Faktoren, die für ein gutes Lernen und eine Beteiligung an der Gesellschaft ausschlaggebend sind. Das Instrument liefert den Schulen, den Kommunen und der Landespolitik steuerungsrelevantes Wissen. Es liefert Lehrern, Eltern, Wissenschaftlern und Politikern Informationen über die sozialen Welten und das Wohlbefinden von Kindern, aus denen sich Angebote entwickeln lassen. Mit UWE gesammelte Informationen werden dabei helfen, die bildungspolitischen und kommunalen Anstrengungen um die Gesundheit und das Wohlbefinden junger Menschen zu verbessern.

UWE sucht Antworten auf drei Hauptfragen:

- Beschreibung: Welches Niveau erreicht das Wohlbefinden der Kinder und Jugendlichen? Wir beschreiben das Niveau und die Streuung des Wohlbefindens aller Siebt- und Neuntklässler in Herne. Unter der Annahme „Durchschnitt

3 Vgl. http://earlylearning.ubc.ca/mdi/ (05.03.2019). 
ist nirgends" werden Unterschiede zwischen Schultypen einerseits und zwischen Sozialräumen andererseits kenntlich gemacht.

- Erklärung: Was brauchen Kinder, damit sie sich wohlfühlen und zu sozial kompetenten und partizipationsfähigen Bürgern entwickeln? Wir erforschen die Ursachen des Wohlbefindens und arbeiten schützende und stärkende Faktoren heraus. Ein besonderer Fokus liegt dabei auf den Kontexten Familie, Schule und Wohnumgebung.

- Transfer: Wie kann Kindern in ihren konkreten Kontexten geholfen werden, ein gesundes Wohlbefinden zu entwickeln? Die Daten haben einen konkreten Praxisbezug hinsichtlich der Unterstützung bei der Planung kleinräumiger und institutionenscharfer Maßnahmen. Wir wollen datengestützt herausstellen, an welchen Ursachen in spezifischen Schulen und Sozialräumen anzusetzen ist, um zu höherem Wohlbefinden der Kinder beizutragen.

Eine vierte Aufgabe ist die Evaluation. Erst mit dieser Aufgabe kommt das gesamte Potenzial des MonitoringInstruments UWE zum Tragen. Dies ist allerdings nur mit einem wiederholten Einsatz des Instruments möglich. Mit einem längerfristigen, kontinuierlichen Monitoring lassen sich mittelfristige und langfristige Veränderungen im Wohlbefinden der Kinder und Jugendlichen verfolgen.

Die Schließung der Informationslücke sollte mit einer Kommune und mit Akteuren begonnen werden, die im Sinne der politischen Ziele der Studie motiviert und im Sinne der handwerklichen Anforderungen fähig sind. Um die Erfolgsbedingungen und die Schwierigkeiten einer möglichen Übertragung des kanadischen Instruments am praktischen Beispiel zu klären, wurde deshalb für Gespräche vor Ort die Stadt Herne ausgewählt. Herne verwendet seit einigen Jahren KOMPIK, die deutsche Adaptation des „Early Development Instrument" (EDI) der University of British Columbia, und die in diesem Zusammenhang von der Bertelsmann Stiftung entwickelte Software „KECK-Atlas“ zur kleinräumigen Dokumentation und Analyse kindlicher Entwicklungsverläufe und ihrer Bedingungen. ${ }^{4}$ Die Stadt ist eine der 40 Modellkommunen im Bundesprogramm „Lernen vor Ort" gewesen und hat die dort entwickelten Handlungsansätze konsequent verstetigt. ${ }^{5}$ Deshalb gibt es in Herne ein kompetentes kommunales Bildungsbüro und

4 Vgl. https://www.keck-atlas.de/keck/teilnehmen/keck-mit-kompik. html (04.03.2019).

5 Vgl. https://www.transferinitiative.de/129.php (04.03.2019). eine sehr gut entwickelte Kooperationskultur zwischen den Akteuren im Bildungsgeschehen. Darüber hinaus ist Herne eine arme Kommune. Die dort gemachten Erfahrungen können deshalb glaubhaft in Empfehlungen an andere Kommunen in finanziell und strukturell ähnlicher oder besserer Lage münden. Im kommunalen Familienbericht werden die Stadt und ihre Sozialstruktur als typisch für den armen Norden des Ruhrgebiets beschrieben (vgl. ZEFIR 2008). Es ist zweckmäßig, bei der Einführung von UWE mit Kommunen zu beginnen, die nicht bei Null anfangen müssen und deren kommunale Spitzen hinter dem Projekt und seinen Zielen stehen.

\section{Theoretischer Hintergrund}

\subsection{System- und Sozialintegration}

Wir verstehen das kommunale kleinräumige Monitoring-Instrument UWE als einen Baustein integrierender Stadtentwicklung. Damit ist gemeint, dass auf der Grundlage der Daten Maßnahmen geplant werden können, die Städte in sozialpolitischer Hinsicht zu konfliktarmen, kohäsiven und solidarischen und damit integrierenden Stadtgesellschaften weiterentwickeln. Der Integrationsaspekt solcher Stadtgesellschaften kann auf zweifache Weise gelesen werden, einerseits als Systemintegration verschiedener Sozialräume und andererseits als Sozialintegration von Kindern und Jugendlichen in die Stadtgesellschaft (vgl. Esser 2001 zu System- und Sozialintegration).

Der Begriff Systemintegration bezieht sich speziell für das UWE-Instrument auf die Eingliederung der Sozialräume und der Schulen in die Stadtgesellschaft. Systemintegration wird unter anderem über die materiellen Interdependenzen der Akteure auf Märkten vermittelt. Beispielsweise wird über den Wohnungsmarkt die Platzierung von Familien im Stadtraum reguliert. Ungleich verteilter Wohnraum (Angebote) und unter Familien ungleich verteiltes Einkommen (Nachfrage) führen zu residentieller Segregation, die sich zumeist als soziale, demographische und ethnische Segregation manifestiert. Ähnliches gilt für die Bildungssegregation. Das Ausmaß der Wohnort- und Bildungssegregation in einer Stadt ist damit ein Indikator für Systemintegration und somit für gelungene Stadtentwicklung oder auch nicht.

Unter Sozialintegration ist der Einbezug der Kinder und Jugendlichen in die Stadtgesellschaft zu verstehen. Zwei Dimensionen stehen im Fokus des UWE-Instruments: Kulturation und Interaktionen. Kulturation bedeu- 
tet, dass die Kinder und Jugendlichen im persönlichen Entwicklungsprozess Wissen, Fertigkeiten und Qualifikationen erwerben. Unter Interaktionen ist die Aufnahme von sozialen Beziehungen im alltäglichen Bereich zu verstehen. Für Kinder und Jugendliche sind das hauptsächlich Beziehungen zur Familie und zu Freunden, aber auch zu Lehrern, zu Übungs-/Kursleitern oder zu Nachbarn. Das Wohlbefinden von Kindern und Jugendlichen ist ein Zustand, der letztlich das Ausmaß der Sozialintegration anzeigt.

Zwischen Sozial- und Systemintegration besteht insofern eine Beziehung, als dass Sozialintegration deutlich besser gelingt, wenn die Systemintegration gelungen ist: Die Kinder und Jugendlichen können sich in ihrer Entwicklung nur entfalten, wenn die sozialräumlichen Kontexte, wozu unter anderem die Schulen und die Wohnumgebungen gehören, weniger stark segregiert sind.

Für die Sozialintegration ist Humanvermögen unerlässlich (vgl. Strohmeier/Gehne/Bogumil et al. 2016). Damit sind soziale und kognitive Fähigkeiten und Kompetenzen gemeint. Vom Ausmaß, in dem Kinder Humanvermögen entwickeln, wird ihre gesellschaftliche, politische und wirtschaftliche Teilhabe abhängen: Gesundheit, Solidarität, Empathie, Vertrauen und Leistungsbereitschaft. Die Erfahrungen von Kindern und Jugendlichen haben entscheidende und langanhaltende Folgen für ihr späteres Leben. Das Wohlbefinden während der Kindheit und Jugend hat einen starken Einfluss auf die Persönlichkeitsentwicklung, die Integrations- und Partizipationsfähigkeit in vielen gesellschaftlichen Bereichen im Erwachsenenalter und damit generell auf die späteren Lebenschancen. Während dieser Zeit erleben sie signifikante kognitive, soziale und emotionale Veränderungen, die ihre Identität bis ins Erwachsenenalter prägen. Das Wohlbefinden von Kindern und Jugendlichen beeinflusst wichtige Fähigkeiten und Kompetenzen, wie sich zu konzentrieren, zu lernen, Freundschaften und andere soziale Kontakte zu entwickeln und zu pflegen sowie überlegte Entscheidungen zu treffen.

Jugendliche haben eine erhöhte Wahrnehmung von sich selbst und von anderen. Sie entwickeln Vorstellungen und Ideen, wie und ob sie in ihre soziale Umwelt passen. Diese Vorstellungen können für die Gesundheit und den schulischen Erfolg förderlich sein oder aber zu negativen Ergebnissen wie Depressionen und Ängsten im Erwachsenenalter führen. Die Jugend ist eine Zeit der Risiken und ebenso eine Zeit der Möglichkeiten. Ernährung und Schlaf, Schulerfahrungen, positive Beziehungen zu Erwachsenen und Gleichaltrigen und organisierte Freizeitaktivitäten in dieser kritischen Zeit sind wichtige Ressourcen für Resilienz und Integrationsfähigkeit der Jugendlichen (Thomson/Oberle/Gadermann et al. 2017). Nicht nur die Familie und die Wohnumgebung sind für die Entwicklung der jungen Gesellschaftsmitglieder wichtige Kontexte. Auch die Schule ist ein Raum für soziale Beziehungen und Erfahrungen, die die Persönlichkeit und ein gelingendes, das heißt sozialintegratives Leben prägen.

\subsection{Kontextabhängigkeit des Wohlbefindens}

Um die Kontextabhängigkeit des Wohlbefindens von Kindern und Jugendlichen zu modellieren, beziehen wir uns auf die Einbettung in Umweltkontexte, wie sie bereits bei Bronfenbrenner (1981) herausgearbeitet wurden (vgl. Abbildung 1). Hiernach ist jedes Kind in eine Reihe von Umweltkontexten eingebettet, die sowohl mit ihm als auch untereinander interagieren und auf diese Art und Weise Einfluss auf seine Entwicklung nehmen. Der das Kind unmittelbar umgebende Kontext (Mikrosystem) ist die Familie. Sie steuert nach Kaufmann, Herlth und Strohmeier (1980) zugleich maßgeblich den Einfluss der weiteren Kontexte. Das heißt, Aufgabe und Leistung der Familie im Sozialisationsprozess ist die Steuerung und Vermittlung von Umwelteinflüssen auf das Kind. Kaufmann, Herlth und Strohmeier (1980) nennen diese Leistung Erziehung.

Die Wohnumgebung, die engere Nachbarschaft und die örtlichen Betreuungs- und Bildungseinrichtungen sowie die sich daraus ergebenden Kontakte zu anderen Erwachsenen und Gleichaltrigen wirken prägend auf die Entwicklung der Kinder und Jugendlichen. Diese Einflüsse ergeben sich mit zunehmendem Alter immer mehr auch ohne die Vermittlung der Familie.

Je älter die Kinder werden, desto wahrscheinlicher sind direkte Effekte räumlicher, sozialer und infrastruktureller Kontexte. In unseren Städten und Gemeinden gibt es eine erhebliche residentielle Segregation sowie bauliche und infrastrukturelle Disparitäten (vgl. Strohmeier 2010). Unterschiedliche kleinräumige und institutionelle Settings und lokale Gemeinschaften haben fördernde oder deprivierende Effekte auf Kinder und Jugendliche. Alle präventiven und kurativen kommunalen Angebote und Dienste wirken unter den Bedingungen der sozialräumlichen Kontexte (vgl. Strohmeier/Gehne/Groos et al. 2014).

Die Entwicklung von Kindern und Jugendlichen wird also von einer Vielzahl von Kontextfaktoren beeinflusst und muss stets im materiellen, sozialen und kulturellen 

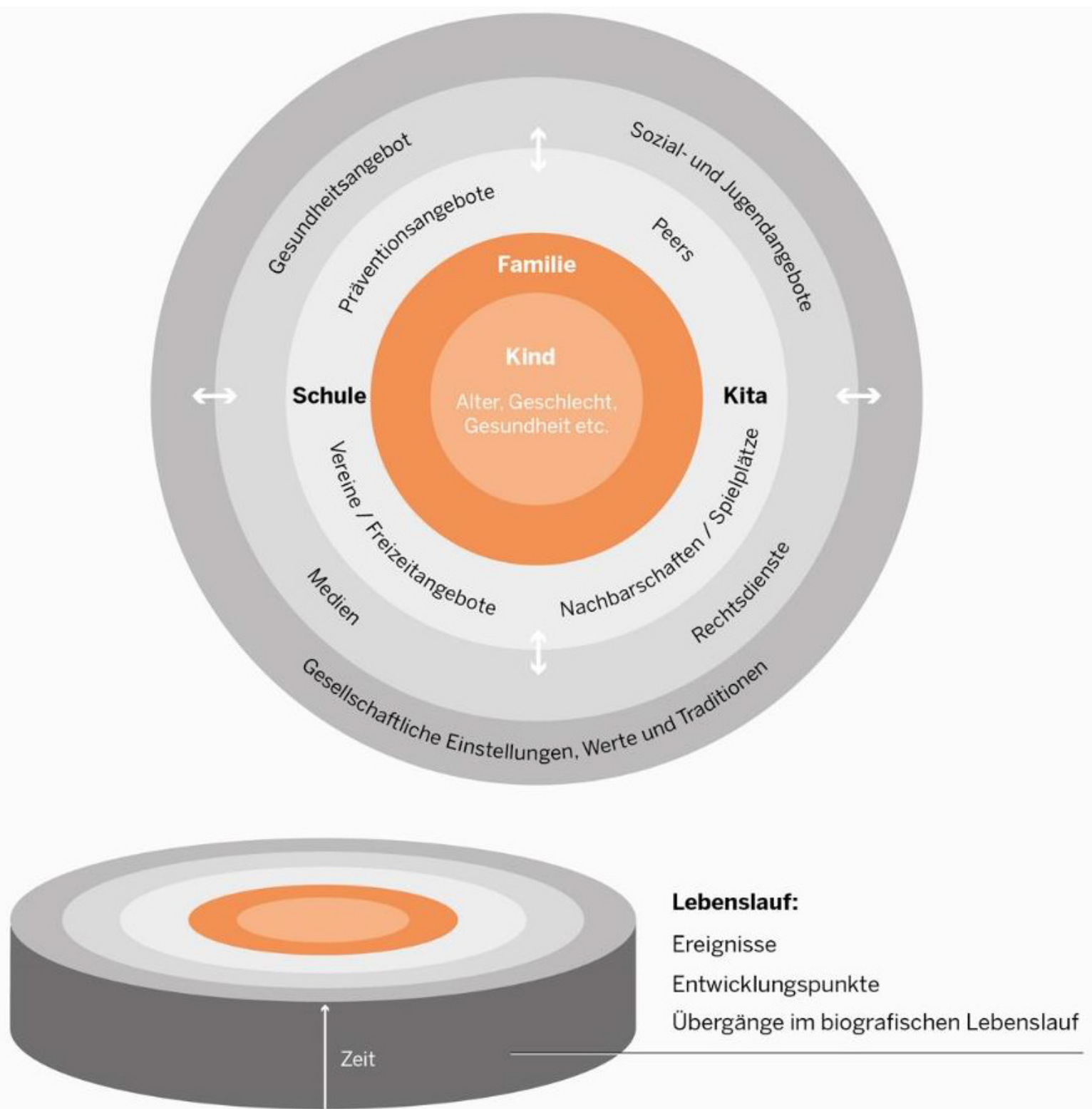

Abbildung 1: Die Ökologie der menschlichen Entwicklung nach Urie Bronfenbrenner. Quelle: Strohmeier/Gehne/Groos et al. (2014: 10)

Kontext betrachtet werden (vgl. Bronfenbrenner 1972; Kaufmann/Herlth/Strohmeier 1980; Bronfenbrenner 1981). Der spezifische Raumbegriff dieses Beitrags orientiert sich also an den sozialräumlichen Kontexten, in denen Kinder und Jugendliche heranwachsen. Zwar identifizieren wir Familie, Schule und Wohnumgebung als bedeutungsvolle Kontexte bzw. informelle Lernorte. Diese sind aber relational im Sinne von Beziehungen zu gleichaltrigen und erwachsenen Mitmenschen geprägt und stellen sich für jedes Kind individuell dar.

Kinder und Jugendliche in ihrer Entwicklung nachhaltig zu fördern bedeutet, die dauerhafte Umwelt zu verändern und zu gestalten, in der sie aufwachsen und alltäglich leben. Dazu muss man verstehen, wie sie wirkt. Für die Beobachtung der frühkindlichen Entwicklung bis zum Ende des fünften Lebensjahres gibt es bereits äquivalente Instrumente und Lösungen in Deutschland. Die Auswertung der Daten der Schuleingangsuntersuchungen oder der von der Bertelsmann Stiftung entwickelte „KOMPIK“ (vgl. Groos 2015; Groos/Jehles 2015) ${ }^{6}$ sind solche Äquivalente. Für das Instrument, das die mittleren Altersjahre in den Vordergrund stellt, gibt es in Deutschland noch kein Äquivalent.

$\overline{6 \quad \text { Vgl. }}$ https://www.keck-atlas.de/keck/teilnehmen/keck-mitkompik.html (04.03.2019). 


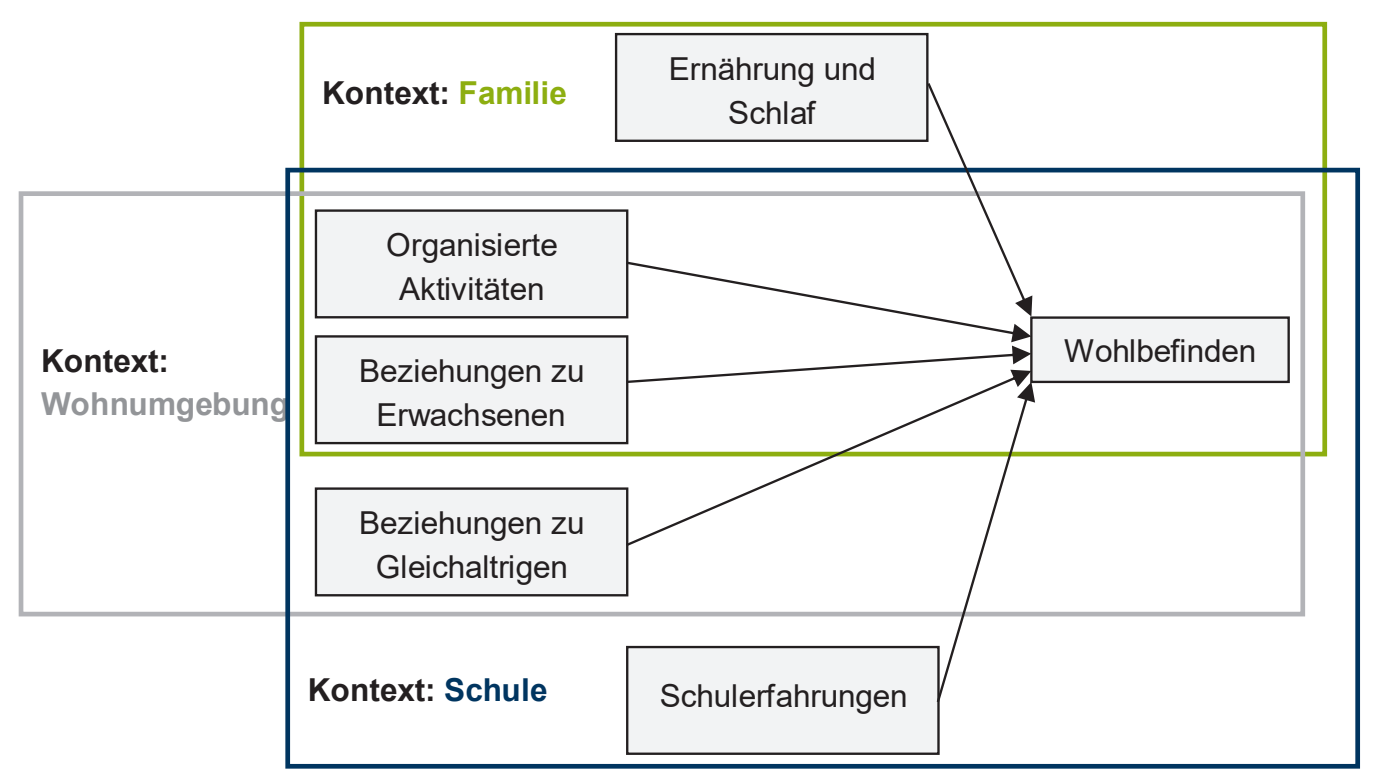

Abbildung 2: Theoretischer Rahmen mit sozialräumlichen Kontexten, Ressourcen und Wohlbefinden

\subsection{Ein Modell des Wohlbefindens von Kindern und Jugendlichen}

Wohlbefinden ist angesagt - nicht nur als Werbeträger für beispielsweise Wohlfühltees oder als therapeutische Anwendungen, die das Wohlbefinden steigern sollen, sondern auch als Forschungsgegenstand (Hascher 2004). Die Erforschung der Entwicklung und des Wohlbefindens von Kindern und Jugendlichen ist in den letzten Jahren immer populärer geworden. Gerade im sozialräumlichen Kontext "Schule" wurden viele Untersuchungen durchgeführt. Die Sonderauswertung Resilienz der PISA-Studie beschreibt beispielsweise die Wichtigkeit eines guten Schulklimas (OECD 2018). Kullmann, Geist und Lütje-Klose (2015) untersuchen das schulische Wohlbefinden in inklusiven Schulen und Hübner, Wagner, Kramer et al. (2017) befassen sich mit Wohlbefinden vor und nach der G8-Reform.

Das Wohlbefinden von Kindern und Jugendlichen wird durch Faktoren gestärkt, die wir Ressourcen nennen. Das Wohlbefinden wird zum Beispiel durch einen gesunden, von ausreichend Ernährung und Schlaf ${ }^{7}$ geprägten Lebenswandel, durch intakte soziale Beziehungen zu Gleichaltrigen und Erwachsenen, durch positive Schulerfahrungen und durch die Einbindung in organisierte Freizeitaktivitäten wie Musik- und Kunstunterricht,

7 Regelmäßige Energieaufnahme und Regeneration sind physiologische Grundvoraussetzungen für die Entwicklung und damit das Wohlbefinden von Menschen.
Sportinitiativen, Jugendgruppen oder Nachhilfeunterricht gestärkt (Schonert-Reichl/Guhn/Gadermann et al. 2012). Die wichtigen sozialräumlichen Kontexte sind im Wesentlichen die Familie, die Schule und die Wohnumgebung. Ressourcen und sozialräumliche Kontexte sind dabei auf je spezifische Weise miteinander verwoben. Die drei Kontexte greifen durch ihre spezifischen Strukturen und Zusammensetzungen in die Verfügbarkeit je spezifischer Ressourcen ein und beeinflussen damit das Wohlbefinden der Heranwachsenden. Die Zusammenhänge sind schematisch in Abbildung 2 dargestellt.

Im Kontext Familie werden drei Ressourcen vermittelt: Ernährung und Schlaf, organisierte Freizeitaktivitäten und Beziehungen zu Eltern und anderen Verwandten bzw. Familienangehörigen. Im Kontext Schule werden vier Ressourcen zur Verfügung gestellt. Hier werden alltägliche Schulerfahrungen gesammelt, Beziehungen zu Gleichaltrigen gestaltet, Beziehungen zu erwachsenen Bezugspersonen etabliert, und Schule ist der Ort von organisierten Aktivitäten am Nachmittag (Arbeitsgruppen oder fakultative Bildungsangebote). Schließlich ist die Wohnumgebung der Kontext, in dem ein großer Teil der Freizeit verbracht wird. Auch hier sind organisierte Aktivitäten in Jugend- und Freizeiteinrichtungen als Ressource zu nennen. Stark prägend ist die Wohnumgebung aber auch als Ort informeller Freizeitaktivitäten, die mit in der Nähe wohnenden, gleichaltrigen Freunden vollzogen werden. Die Wohnumgebung ist zudem wichtig für die Sozialisation: Insbesondere erwachsene 
Bewohner - nicht nur Nachbarn - sind Rollenvorbilder für Kinder und Jugendliche.

Wovon hängt das Wohlbefinden der Kinder und Jugendlichen ab? Welche Rolle spielen unterschiedliche Schulformen im gegliederten Schulsystem? Welche Rolle spielen sozialräumliche Kontextfaktoren?

Hypothese 1: Je mehr Ressourcen den Kindern und Jugendlichen zur Verfügung stehen, desto größer ist die Wahrscheinlichkeit für ein positives Wohlbefinden.

Im Jahr 2005 hat UNICEF einen mehrdimensionalen Ansatz zum kindlichen Wohlbefinden entwickelt (vgl. Bertram/Stein/Niejahr 2011). Materielles Wohlbefinden, Gesundheit und Sicherheit, Bildung und Ausbildung, Beziehungen zu Gleichaltrigen und zur Familie, Verhaltensrisiken und subjektives Wohlbefinden standen dabei im Fokus und bezogen sich unmittelbar auf den 41. Artikel der UN-Kinderrechtskonvention. ${ }^{8}$ Bertram, Stein und Niejahr (2011) beschreiben die Bedeutung dieser Ressourcen für das Wohlergehen und bemängeln zugleich, dass es zwar bereits zahlreiche Initiativen gebe, die sich mit kindlichem Wohlbefinden auseinandersetzen, dass diese sich jedoch immer nur auf einzelne Dimensionen beziehen. Eine ganzheitliche Betrachtung und das abgestimmte Zusammenwirken der Akteure vor Ort sei dafür dringend erforderlich. Zudem zeigt das LBS-Kinderbarometer, wie wichtig die Abfrage der Stimmungen und Meinungen von Kindern ist (Beisenkamp/Klöckner/Hallmann et al. 2009).

Hypothese 2: Die Ressourcenausstattung im Zusammenhang mit dem Wohlbefinden unterscheidet sich bei alleinerziehenden Eltern kaum von Paarhaushalten.

Eine Untersuchung des Bundesministeriums für Familie, Senioren, Frauen und Jugend zeigte, dass Kinder alleinerziehender Mütter nur unwesentlich weniger Ressourcen zur Verfügung haben als Jugendliche, auf die dies nicht zutrifft (BMFSFJ 2012: 21). Obwohl Kinder aus Paarfamilien günstigere Entwicklungsmilieus aufweisen als Jugendliche aus Ein-Eltern-Familien, beschreiben auch Walper und Wendt (2005) eher schwache Effekte. Signifikante Unterschiede in der Ressourcenausstattung sind somit nicht zu erwarten.

Hypothese 3: Das Wohlbefinden zeigt schulspezifische Unterschiede auf.

„Wohlbefinden resultiert vor allem aus der Qualität der sozialen Beziehungen (Akzeptanz durch die Lehrer und Integration bei den Mitschülern), aus der Bedeutsamkeit und Schülerzentriertheit des Unterrichts, aus der Zufriedenheit mit der eigenen Leistung und einem

8 Vgl. https://www.kinderrechtskonvention.info/kinderrechtskonvention-352/ (05.03.2019). positiven Klima der Schule" (Eder 2004: 92). Dalbert und Stöber (2005) verdeutlichen, dass Schüler, die sich von ihren Lehrern gerecht behandelt fühlen, auch ein höheres schulisches Wohlbefinden an den Tag legen. Diese gerechte Behandlung erleben nach Meister-Wolf (2003) Ganztagsschüler häufiger als Halbtagsschüler. Da Kinder und Jugendliche in Ganztagsschulen einen längeren Zeitraum miteinander verbringen, ist das Streitund Konfliktpotenzial höher und das Sozialklima fällt dadurch oft schlechter aus (Meister-Wolf 2003: Abschnitt 10.1 o.S.). So zeigen sich schulspezifische Unterschiede, die sich auch im Wohlergehen auswirken.

Hypothese 4: Die Wohnumgebung hat einen Einfluss auf das Wohlbefinden.

Studien zeigen, dass die Wohnumgebung einen Einfluss auf das Wohlbefinden ausübt. Der British Columbia Atlas of Child Development beschreibt, wie höhere Risikolagen in Bezug auf die körperliche Gesundheit von Jugendlichen in einem Sozialraum mit einem hohen Anteil an Geringverdienern zusammenhängt (Kershaw/ Irwin/Trafford et al. 2005). Auch von der Zusammensetzung der direkten Wohnumgebung, der Nachbarschaft, kann das Wohlbefinden abhängen (BMFSFJ 2015: 45). Zudem kann gleichermaßen das äußere Erscheinungsbild des Quartiers (z. B. Verschmutzung, Beschädigung von Sitzmöglichkeiten) die Beurteilung des Wohlbefindens beeinflussen (Hilke 2013: 58 ff.). Nicht zuletzt spielen die in der direkten Wohnumgebung vorhandenen Ressourcen eine wichtige Rolle für das Wohlbefinden.

\section{Methodisches Vorgehen}

Da die UWE-Studie eine Adaption des kanadischen MDI ist, wurde im ersten Schritt der kanadische Fragebogen übersetz $t^{9}$ und an das nordrhein-westfälische Schulsystem angepasst. Die Anpassung geschah im Rahmen eines Workshops mit dem Bildungsbüro der Stadt Herne und Vertretern der beteiligten weiterführenden Schulen. Daraufhin wurde der überarbeitete Fragebogen mit

9 Die deutsche Übersetzung wurde in Zusammenarbeit zwischen der Human Early Learning Partnership (HELP) der University of British Columbia, dem Mannheimer Institut für Public Health (MIPH) der Universität Heidelberg und dem Zentrum für Interdisziplinäre Regionalforschung (ZEFIR) der Ruhr-Universität Bochum erstellt. Sie wurde mit Mitteln des Wissenschaftsministeriums des Landes Baden-Württemberg unter der Sonderlinie Medizin im Projekt „Ein gutes Jahr mehr für jeden Bürger“ (MIPH) und des Forschungsinstituts für gesellschaftliche Weiterentwicklung des Landes Nordrhein-Westfalen im Projekt „Wie erleben Kinder sozialräumliche Segregation und Bildungssegregation“ gefördert. 
Herner Schülern der Klassen 6 und 8 in Fokusgruppen und Pretests diskutiert und getestet. Datenschutzbeauftragte der Schulen und der beteiligten Institutionen prüften den Fragebogen und die begleitenden Materialien auf datenschutzrelevante Belange. Die Durchführung der Auswertung wurde seitens des Zentrums für interdisziplinäre Regionalforschung (ZEFIR), welches mit der Erhebung, Analyse und Auswertung der Daten beauftragt ist, getestet. Alle bis dahin erhaltenen Ergebnisse wurden wiederum mit dem Bildungsbüro und den weiteren beteiligten Akteuren diskutiert.

Dann wurde die Befragung an allen öffentlichen weiterführenden Schulen in Herne durchgeführt, die eine Mittelstufe haben. Die Studie wurde als Vollerhebung realisiert, das heißt, dass alle Kinder und Jugendlichen, die die siebte oder neunte Klasse besuchten, die Möglichkeit hatten, an der Befragung teilzunehmen. Diese Art der Erhebung ist notwendig, da das Monitoring-Instrument institutionenscharfe Ergebnisse verarbeiten soll. Es haben 14 Schulen an UWE teilgenommen, darunter zwei Hauptschulen, vier Realschulen, drei Gesamtschulen und fünf Gymnasien. Die Grundgesamtheit bilden alle 2.838 schulpflichtigen Siebt- und Neuntklässler. Es nahmen insgesamt 1.962 Interessierte teil, sodass ein Rücklauf von rund $69 \%$ erzielt wurde..$^{10}$ Die Kommunikationsstrukturen in den Schulen, beispielsweise zwischen Schulleitung und Lehrerkollegium oder zwischen Lehrern und Schülern, hatten Einfluss auf die Anzahl der abgegebenen Elterneinverständniserklärungen und damit auf die selektive Beteiligung der Kinder. In Nordrhein-Westfalen setzt die Befragung Minderjähriger (hier zwischen 12 und 16 Jahren) die Einverständniserklärung der Eltern voraus. So wurden neben den Schulen auch die Erziehungsberechtigten der Kinder und Jugendlichen über das Vorhaben informiert. Die unterschriebenen Einverständniserklärungen wurden von den Klassenlehrern eingesammelt und diese führten zugleich eine Liste der zur Teilnahme berechtigten Kinder. Diese Liste wurde von den am Tag der Befragung unterrichtenden Lehrern vorgelesen, sodass sich die Kinder und Jugendlichen, welche einen Fragebogen ausfüllen durften, melden konnten. Nur an diese wurden die Fragebögen ausgeteilt. Kinder, die nicht an der Befragung teilnehmen durften, bekamen Ersatzaufgaben von ihren Lehrern, die sie, je nach zur Verfügung stehenden Räumlichkeiten und Aufsichtspersonen, entweder im Klassenverband lösten oder in einem separaten Klassenzimmer bearbeiteten. Um Absprachen und eine gegenseitige Beeinflussung der Kinder und Jugendlichen zu vermeiden, wurden alle Teilnehmer einer Jahrgangsstufe in einer Schule zeitgleich befragt. Die Befragung dauerte durchschnittlich 60 Minuten. Dabei wurden die Siebtklässler, sofern dies möglich war, unmittelbar vor den großen Pausen befragt, sodass innen bei einer möglicherweise längeren Bearbeitungszeit noch die Pausenzeit zum Ausfüllen der Bögen zur Verfügung stand. Die Neuntklässler, welche in der Regel eine kürzere Bearbeitungszeit benötigten, wurden im Anschluss ebenfalls im Klassenverband und stufenweise befragt.

Die Befragung wurde anonym und anhand von Papierfragebögen durchgeführt. Nach der Bearbeitung einer gemeinsam besprochenen Einführungsseite, auf der die Kinder und Jugendlichen die verschiedenen Fragetypen und Ankreuzmöglichkeiten kennenlernten, antworteten diese auf 21 Fragen mit 147 Items, die zum Teil in Fragebatterien gestellt wurden. Den Teilnehmern war es während der gesamten Befragung möglich, den Interviewern Rückfragen zu den einzelnen Items zu stellen. Sobald die Kinder und Jugendlichen den Bogen ausgefüllt hatten, suchten diese auf einem Stadtplan von Herne, welcher an der Tafel hing, ihren Wohnort in einem Straßenverzeichnis. Jede mit einem Buchstaben und einer Zahl gekennzeichnete Straße konnte so über ein Koordinatensystem auf der Karte gefunden werden. Die Kinder und Jugendlichen nannten den Interviewern die Zahl des statistischen Bezirks, innerhalb dessen sie wohnten. So konnten diese nicht nur den Schulformen, sondern auch den statistischen Bezirken ihrer Wohnorte in Herne zugeordnet werden. Die ausgefüllten Fragebögen wurden in einer Versandtasche gesammelt und versiegelt, bevor diese in einem Druckzentrum automatisiert eingelesen und damit digitalisiert wurden. Anschließend wurden auch die handschriftlich ausgefüllten Felder anonymisiert und der Text somit ebenfalls digitalisiert, sodass keine Rückschlüsse auf die Identität der Kinder und Jugendlichen möglich und die Datenschutzrichtlinien für Minderjährige gewährleistet sind.

10 Unter der Prämisse, möglichst alle Kinder und Jugendlichen in einer Kommune zu befragen, ist der Zugang über die Schulen und die Befragung im Klassenverband forschungsökonomisch am sinnvollsten. In der Rücklaufquote kommt zum Ausdruck, dass Schulverweigerer ebenso wie am Befragungstag kranke oder verhinderte Jugendliche nicht erfasst werden, aber auch, dass Elterneinverständnisse am Befragungstag nicht vorlagen. 


\section{Analysen}

\subsection{Wohlbefinden und stärkende Ressourcen}

Das Wohlbefinden von Kindern und Jugendlichen ist das Resultat kognitiver Erfahrungen und emotionaler Erlebnisse. Es wird als Vorhandensein von positiven Emotionen und Kognitionen sowie als Abwesenheit von negativen Emotionen, Kognitionen und Beschwerden definiert. Die vorliegende Studie arbeitet mit fünf Konstrukten der sozialen, emotionalen und gesundheitlichen Entwicklung: Optimismus, Selbstwertgefühl, Glücksempfinden, Abwesenheit von Traurigkeit und der allgemeine Gesundheitszustand. Die Konstrukte setzen sich zumeist aus mehreren Items zusammen. Für jedes Konstrukt wird eine Skala mit drei Ausprägungen (niedrig, mittel, hoch) erzeugt. Der Index des Wohlbefindens basiert auf diesen fünf Skalen (vgl. Abbildung 3). Niedriges Wohlbefinden ( $53 \%$ der befragten Kinder) bedeutet, dass bei mindestens einer der fünf Skalen eine niedrige Ausprägung vorliegt. Hohes Wohlbefinden (16\%) setzt hohe Ausprägungen in mindestens vier Skalen voraus. Gibt es weniger Skalen mit hoher Ausprägung und keine Skala mit einer negativen Ausprägung, wird das Wohlbefinden als mittel (31\%) klassifiziert. Die Indexbildung produziert also kein Abbild des durchschnittlichen Wohlbefindens, sondern ein asymmetrisches. Im niedrigen Wohlbefinden sind nicht nur extreme Fälle konzentriert, es stellt vielmehr das breite Potenzial für Verbesserungen des Wohlbefindens dar.

Im theoretischen Konzept wurden fünf wichtige Ressourcen identifiziert, die den Kindern und Jugendlichen helfen, positive Entwicklungen zu nehmen und ihr Wohlbefinden zu fördern (vgl. Abbildung 1). Für die Ressource Ernährung und Schlaf wurde erhoben, an wie vielen Tagen einer normalen Woche gefrühstückt, mit Erwachsenen eine Mahlzeit eingenommen und gut geschlafen wird. Die Ressource Schulerfahrungen bezieht sich auf den respektvollen, unterstützenden sozialen Umgang an der Schule sowie das Mobbingverhalten. Mit Beziehungen zu Erwachsenen ist gemeint, dass in der Familie, der Schule und der Nachbarschaft jeweils eine erwachsene Person existiert, die das Kind ernstnimmt, inm zuhört und es wichtig nimmt. Beziehungen zu Gleichaltrigen basieren dagegen auf einem Gefühl der Gruppenzugehörigkeit und engen Freundschaften. Schließlich wurde für die Ressource organisierte Freizeitaktivitäten abgefragt, an wie vielen Tagen einer normalen Woche organisierte Freizeitaktivitäten wahrgenommen werden. $\mathrm{Da}$ sich die fünf Ressourcen in der Anzahl zugrunde

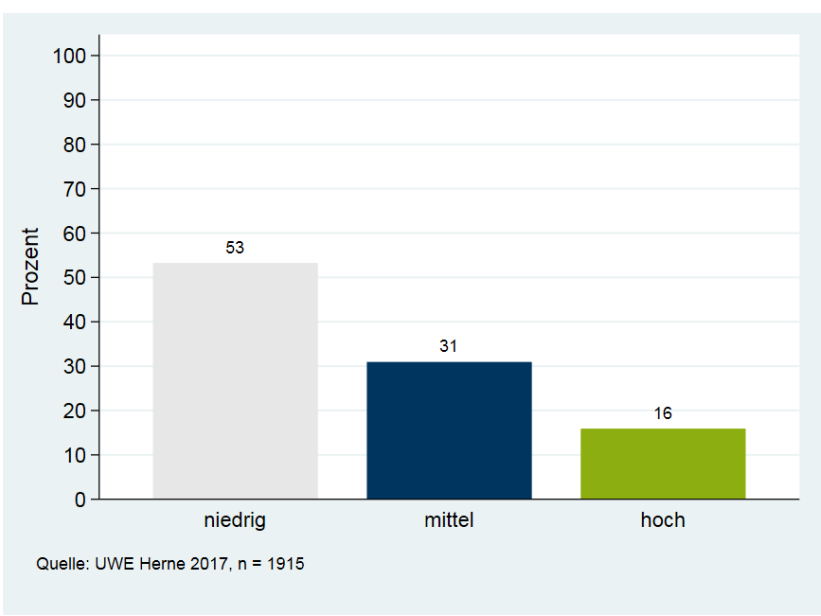

Abbildung 3: Verteilung des subjektiven Wohlbefindens von Kindern und Jugendlichen

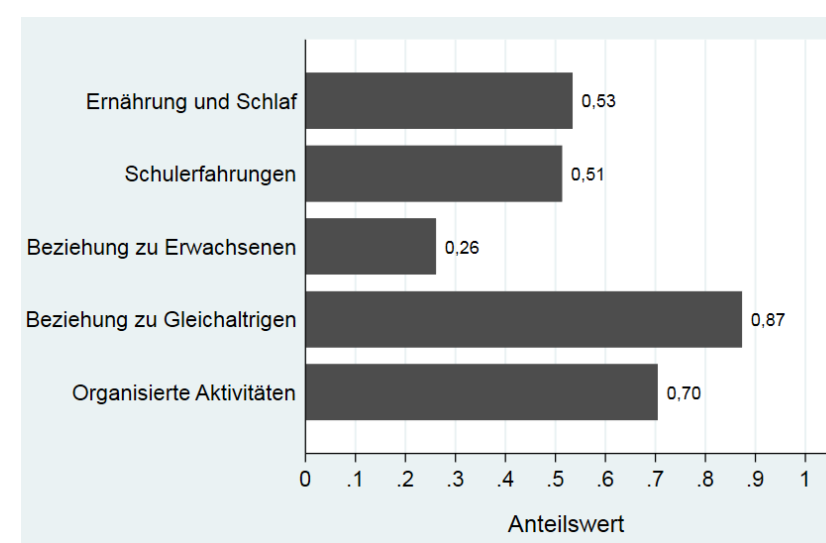

Quelle: UWE Herne 2017, $\mathrm{n}_{\min }=1878$ und $\mathrm{n}_{\max }=1941$

Abbildung 4: Verfügbarkeit zentraler, entwicklungsrelevanter Ressourcen

liegender Fragen und Antworten unterscheiden, wurde gemessen, ob jede der entwicklungsrelevanten Ressourcen vorhanden ist bzw. zur Verfügung steht. Die fünf Anteilswerte sind in Abbildung 4 dargestellt.

Abbildung 4 ist zu entnehmen, dass Beziehungen zu Gleichaltrigen am weitesten verbreitet sind: $87 \%$ der befragten Kinder und Jugendlichen verfügen über diese Ressource. Ebenfalls eine deutliche absolute Mehrheit geht organisierten Aktivitäten in der Freizeit nach (70\%). Dagegen hat nur jedes vierte Kind (26\%) wichtige erwachsene Bezugspersonen in Familie, Schule und Nachbarschaft. Die Ressourcen sind also recht ungleich verteilt und zeigen durchaus überraschende Befunde. Über die fünf Ressourcen ist ein Summenindex gebildet worden (vgl. Abbildung 5). Nur eine extreme Minderheit (3 \%) verfügt über keinerlei stärkende Ressourcen; 
Tabelle 1: Regressionen des Wohlbefindens auf stärkende Ressourcen

\begin{tabular}{|c|c|c|c|c|c|c|}
\hline Variablen & Modell 1 & & & Modell 2 & & \\
\hline \multicolumn{7}{|l|}{ Ressourcen } \\
\hline Ernährung und Schlaf & 0,865 & $* * *$ & $(8,54)$ & & & \\
\hline Schulerfahrungen & 0,894 & *** & $(8,75)$ & & & \\
\hline Beziehungen zu Erwachsenen & 0,493 & *** & $(4,60)$ & & & \\
\hline Beziehungen zu Gleichaltrigen & 0,761 & *** & $(4,23)$ & & & \\
\hline organisierte Aktivitäten & 0,050 & & $(0,45)$ & & & \\
\hline Summenindex & & & & 0,633 & *** & $(14,52)$ \\
\hline \multicolumn{7}{|l|}{ Kontrollvariablen } \\
\hline Geschlecht (1=weiblich) & $-0,501$ & *** & $(5,15)$ & $-0,473$ & $* * *$ & $(4,90)$ \\
\hline Alter & $-0,121$ & ** & $(3,06)$ & $-0,099$ & ** & $(2,58)$ \\
\hline Migrationshintergrund (1=vorhanden) & 0,132 & & $(0,10)$ & 0,167 & & $(0,10)$ \\
\hline \multicolumn{7}{|l|}{ Übergänge im Wohlbefinden } \\
\hline Übergang von niedrig zu mittel & $-0,010$ & & $(0,02)$ & 0,397 & & $(0,69)$ \\
\hline Übergang von mittel zu hoch & 1,746 & ** & $(2,87)$ & 2,125 & $* * *$ & $(3,66)$ \\
\hline \multicolumn{7}{|l|}{ Modellstatistiken } \\
\hline Fallzahl & 1739 & & & 1739 & & \\
\hline McFadden Pseudo- $R^{2}$ & 0,092 & & & 0,081 & & \\
\hline $\mathrm{BIC}$ & 3225,1 & & & 3234,1 & & \\
\hline
\end{tabular}

Alle Modelle sind Ordered-Logit-Regressionen für niedriges (Wert 0), mittleres (1) und hohes (2) Wohlbefinden. Berichtet werden Koeffizienten und in Klammern z-Statistiken. *** $\alpha \leq 0,001 ;{ }^{* *} \alpha \leq 0,01 ;{ }^{*} \alpha \leq 0,05$

weitere $11 \%$ können nur auf eine Ressource zurückgreifen. Mehr als die Hälfte der befragten Kinder hat drei oder vier Ressourcen zur Verfügung.

Mit Regressionsanalysen wird geprüft, ob die Ressourcen tatsächlich das Wohlbefinden stärken. Ein Modell wird mit den fünf Ressourcen geschätzt, ein zweites mit dem Summenindex der Ressourcen. In beide Regressionen gehen das Geschlecht, das Alter und der Migrationshintergrund als Kontrollvariablen ein (vgl. Tabelle 1).

Die erwarteten Zusammenhänge bestätigen sich weitestgehend. Die Ressourcen hängen mit dem Wohlbefinden zusammen, insbesondere Ernährung und Schlaf sowie Schulerfahrungen korrelieren stark mit dem Wohlbefinden. Einzig die Ressource „organisierte Aktivitäten" weist keinen signifikanten Zusammenhang auf. Die zweite Regression zeigt, dass auch der Summenindex über die fünf Ressourcen stark mit dem Wohlbefinden zusammenhängt. Es lässt sich konstatieren, dass ausreichend Ernährung und Schlaf, positive

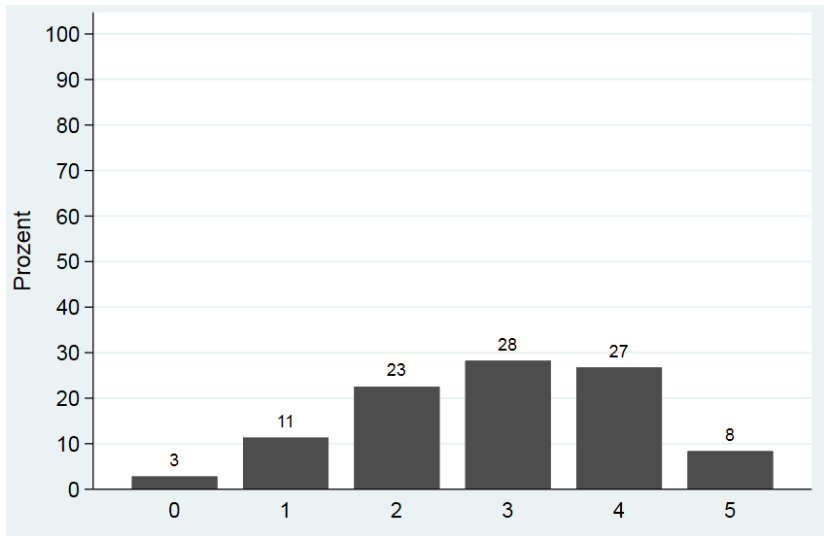

Quelle: UWE Herne 2017, $n=1817$

Abbildung 5: Anzahl der Ressourcen pro Kind

Schulerfahrungen, starke Beziehungen zu Erwachsenen in Familie, Schule und Nachbarschaft sowie zu Gleichaltrigen stärkende Ressourcen für das Wohlbefinden sind. Im Hinblick auf die Kontrollvariablen kann 
Tabelle 2: Regressionen des Wohlbefindens auf stärkende Ressourcen im Familienkontext

\begin{tabular}{|c|c|c|c|c|c|c|c|c|c|}
\hline Variablen & Modell 1 & & & Modell 2 & & & Modell 3 & & \\
\hline \multicolumn{10}{|l|}{ Ressourcen } \\
\hline Ernährung und Schlaf & 0,855 & $* * *$ & $(8,34)$ & & & & 0,795 & $* * *$ & $(7,12)$ \\
\hline Schulerfahrungen & 0,861 & $* * *$ & $(8,34)$ & & & & 0,864 & $* * *$ & $(8,36)$ \\
\hline Beziehungen zu Erwachsenen & 0,471 & *** & $(4,35)$ & & & & 0,500 & $* * *$ & $(4,29)$ \\
\hline Beziehungen zu Gleichaltrigen & 0,758 & $* * *$ & $(4,18)$ & & & & 0,752 & *** & $(4,14)$ \\
\hline organisierte Aktivitäten & $-0,001$ & & $(0,01)$ & & & & 0,070 & & $(0,56)$ \\
\hline Summenindex & & & & 0,607 & $* * *$ & $(13,79)$ & & & \\
\hline \multicolumn{10}{|l|}{ Familienkontext } \\
\hline Alleinerziehenden-Haushalt (AE-Haushalt) & $-0,539$ & $* * *$ & $(3,99)$ & $-0,519$ & $* * *$ & $(3,88)$ & $-0,426$ & & $(1,50)$ \\
\hline Anzahl Geschwister & $-0,048$ & & $(0,92)$ & $-0,031$ & & $(0,59)$ & $-0,045$ & & $(0,86)$ \\
\hline \multicolumn{10}{|l|}{ Ressourcen im Familienkontext } \\
\hline AE-Haushalt * Ernährung und Schlaf & & & & & & & 0,340 & & $(1,23)$ \\
\hline AE-Haushalt * Beziehungen zu Erwachsenen & & & & & & & $-0,244$ & & $(0,79)$ \\
\hline AE-Haushalt * organisierte Aktivitäten & & & & & & & $-0,371$ & & $(1,30)$ \\
\hline \multicolumn{10}{|l|}{ Kontrollvariablen } \\
\hline Geschlecht (1=weiblich) & $-0,510$ & $* * *$ & $(5,18)$ & $-0,478$ & $* * *$ & $(4,90)$ & $-0,507$ & $* * *$ & $(5,15)$ \\
\hline Alter & $-0,118$ & ** & $(2,93)$ & $-0,096$ & * & $(2,45)$ & $-0,116$ & ** & $(2,90)$ \\
\hline Migrationshintergrund ( $1=$ =vorhanden $)$ & 0,121 & & $(1,19)$ & 0,151 & & $(1,50)$ & 0,121 & & $(1,18)$ \\
\hline \multicolumn{10}{|l|}{ Übergänge im Wohlbefinden } \\
\hline Übergang von niedrig zu mittel & $-0,258$ & & $(0,41)$ & 0,190 & & $(0,32)$ & $-0,206$ & & $(0,33)$ \\
\hline Übergang von mittel zu hoch & 1,513 & ** & $(2,43)$ & 1,931 & ** & $(3,25)$ & 1,566 & ** & $(2,50)$ \\
\hline \multicolumn{10}{|l|}{ Modellstatistiken } \\
\hline Fallzahl & 1713 & & & 1713 & & & 1713 & & \\
\hline McFadden Pseudo-R ${ }^{2}$ & 0,097 & & & 0,085 & & & 0,098 & & \\
\hline $\mathrm{BIC}$ & 3177,2 & & & 3188,2 & & & 3196,1 & & \\
\hline
\end{tabular}

Alle Modelle sind Ordered-Logit-Regressionen für niedriges (Wert 0), mittleres (1) und hohes (2) Wohlbefinden. Berichtet werden Koeffizienten und in Klammern z-Statistiken. ${ }^{* * *} \alpha \leq 0,001 ;{ }^{* *} \alpha \leq 0,01 ;{ }^{*} \alpha \leq 0,05$

darüber hinaus festgehalten werden, dass Mädchen ein signifikant geringeres Wohlbefinden aufweisen als Jungen und höheres Alter mit niedrigeren Stufen des Wohlbefindens einhergeht. Die Insignifikanz des Migrationshintergrunds deutet darauf hin, dass sich Kinder mit Migrationshintergrund im Wohlbefinden nicht von Kindern ohne Migrationshintergrund unterscheiden. In den nächsten Analysen werden die drei sozialräumlichen Kontexte Familie, Schule und Wohnumgebung schrittweise berücksichtigt.

\subsection{Familie als sozialräumlicher Kontext des Wohlbefindens}

Hinsichtlich des Familienkontextes wird unterschieden, ob die Kinder und Jugendlichen in einem Alleinerziehenden-Haushalt aufwachsen oder ob mindestens zwei Erwachsene - in der Regel die Eltern - zum Haushalt gehören. Rund 20 \% der befragten Herner Kinder leben in einem Haushalt mit nur einem Erwachsenen, in der Regel ist das die Mutter. Ein zweites Kriterium ist die 
Anzahl der Geschwister. Die überwiegende Mehrheit wächst mit Geschwistern auf (84 \%), wovon $39 \%$ einen Bruder oder eine Schwester, $24 \%$ zwei Geschwister und $21 \%$ drei oder mehr Geschwister haben. Lediglich $14 \%$ sind Einzelkinder.

Modell 1 in Tabelle 2 basiert auf Modell 1 in Tabelle 1 , erweitert dieses aber um die beiden unabhängigen Variablen Alleinerziehenden-Haushalt und Geschwisteranzahl. Es zeigt sich, dass Kinder aus AlleinerziehendenHaushalten ein substanziell geringeres Wohlbefinden aufweisen als Kinder aus anderen Haushalten. Andererseits ist die Anzahl der Geschwister belanglos für das Wohlbefinden. Dieses Muster bestätigt sich, wenn statt der fünf Ressourcen der Summenindex in die Regression einbezogen wird (Modell 2 in Tabelle 2). In Modell 3 der Tabelle 2 sind zusätzlich zu den fünf Einzelressourcen und den beiden Merkmalen des Familienkontextes drei Interaktionsterme zwischen den Ressourcen „Ernährung und Schlaf“, „Beziehungen zu Erwachsenen“ und "organisierte Aktivitäten“ aufgenommen. Diese Interaktionen stellen Unterschiede der Beziehungen zwischen den Ressourcen und dem Wohlbefinden für Alleinerziehenden-Haushalte im Vergleich zu Haushalten mit mehreren erwachsenen Personen dar. Es stellt sich allerdings heraus, dass diese Interaktionsterme keine relevanten Zusammenhänge mit dem Wohlbefinden aufweisen. Zugleich hat sich der Betrag des Koeffizienten für die Alleinerziehenden-Haushalte verkleinert und ist nicht mehr signifikant. Dies kann so gedeutet werden, dass sich der Einfluss des Familienkontexts (Alleinerziehenden-Haushalt) teilweise über die drei spezifischen Ressourcen auf das Wohlbefinden auswirkt, allerdings sind diese Zusammenhänge praktisch irrelevant (nicht signifikant) und verlaufen in unterschiedliche Richtungen: Während der Einfluss von Beziehungen zu Erwachsenen und von organisierten Aktivitäten in Alleinerziehenden-Haushalten tendenziell schwächer ist (negative Interaktionsterme), wird die Beziehung zwischen Ernährung und Schlaf sowie Wohlbefinden für Kinder aus Alleinerziehenden-Haushalten tendenziell gestärkt (positiver Interaktionsterm).

\subsection{Schule als sozialräumlicher Kontext des Wohlbefindens}

Als zweiten Kontext wird die Schule betrachtet. Zunächst werden die verschiedenen Schultypen unterschieden. In die Untersuchung sind zwei Haupt-, vier Real- und drei Gesamtschulen sowie fünf Gymnasien aufgenommen worden (gesamt: 14). Herner Förderschulen und
Privatschulen wurden nicht in die Untersuchung einbezogen; Sekundarschulen gibt es in Herne nicht. Für die Auswertung wurden drei Schultypen untersucht. Hauptund Realschulen zusammen bilden die Referenzkategorie; Differenzen dazu werden für Gesamtschulen und Gymnasien analysiert. Die Analyseergebnisse sind in Tabelle 3 verzeichnet.

In den Modellen 1 und 2 in Tabelle 3 zeigen sich nun etwas überraschende Befunde. So gibt es keinen substanziellen Unterschied im Wohlbefinden der Kinder aus Haupt- und Realschulen einerseits und Gymnasien andererseits. Dagegen haben Kinder, die Gesamtschulen besuchen, ein signifikant höheres Wohlbefinden als Kinder von Haupt- und Realschulen. Das Ergebnis ist robust, denn es wird für beide Regressionen mit Einzelressourcen und mit dem Summenindex der Ressourcen festgestellt. In Modell 3 in Tabelle 3 werden zusätzlich Interaktionsvariablen zwischen Schulkontexten und Einzelressourcen berücksichtigt. Allerdings ist kein einziger Koeffizient der Interaktionsvariablen signifikant. Das bedeutet, dass es keine relevanten schultypspezifischen Unterschiede der Zusammenhänge zwischen Ressourcen und Wohlbefinden gibt. Zugleich ist der Zusammenhang zwischen Gesamtschulen und Wohlbefinden nicht mehr signifikant, obwohl sich der Koeffizient gegenüber Modell 1 verdoppelt hat. Nachvollziehbar wird dies, wenn man berücksichtigt, dass alle vier Interaktionsterme mit der Gesamtschule negativ sind, also tendenziell abgeschwächte Ressourcenzusammenhänge für Gesamtschüler ausweisen. In der Summe ist das Wohlbefinden an Gesamtschulen substanziell höher (unter Berücksichtigung der sozialen Zusammensetzungen an Schulen), einzelne Beträge, die sich auf den Schultyp an sich und die schultypischen Zusammenhänge zwischen Ressourcen und Wohlbefinden zurückführen lassen, sind für sich genommen nicht substanziell unterschiedlich.

Unterschiedliche Schultypen als schulische soziale Kontexte aufzufassen, kann als eine zu grobe Verallgemeinerung angesehen werden. Institutionenscharfen Analysen können auch einzelne Schulen als Kontexte zugrunde liegen. Lassen sich Unterschiede im Wohlbefinden und in den Zusammenhängen von Ressourcen und Wohlbefinden für einzelne Schulen feststellen, wären gezieltere Interventionen in der Praxis möglich. Um dies zu untersuchen, wurden weitergehende, sogenannte Mehrebenenanalysen durchgeführt. Tests zeigen, dass die substanziellen Variationen zwischen den Schulen diese Analysen nicht rechtfertigen. Mit anderen Worten: Es gibt keine systematischen Unterschiede im aggregierten Wohlbefinden zwischen einzelnen Schulen. 
Tabelle 3: Regressionen des Wohlbefindens auf stärkende Ressourcen im Schulkontext

\begin{tabular}{|c|c|c|c|c|c|c|c|c|c|}
\hline Variablen & Modell 1 & & & Modell 2 & & & Modell 3 & & \\
\hline \multicolumn{10}{|l|}{ Ressourcen } \\
\hline Ernährung und Schlaf & 0,861 & $* * *$ & $(8,37)$ & & & & 0,855 & $* * *$ & $(8,27)$ \\
\hline Schulerfahrungen & 0,877 & $* * *$ & $(8,45)$ & & & & 0,821 & $* * *$ & $(4,87)$ \\
\hline Beziehungen zu Erwachsenen & 0,462 & *** & $(4,26)$ & & & & 0,543 & ** & $(3,00)$ \\
\hline Beziehungen zu Gleichaltrigen & 0,744 & $* * *$ & $(4,10)$ & & & & 0,786 & ** & $(2,74)$ \\
\hline organisierte Aktivitäten & 0,020 & & $(0,18)$ & & & & $-0,050$ & & $(0,29)$ \\
\hline Summenindex & & & & 0,618 & $* * *$ & $(13,84)$ & & & \\
\hline \multicolumn{10}{|l|}{ Familienkontext } \\
\hline Alleinerziehenden-Haushalt & $-0,551$ & $* * *$ & $(4,07)$ & $-0,536$ & $* * *$ & $(3,99)$ & $-0,549$ & $* * *$ & $(4,03)$ \\
\hline Anzahl Geschwister & $-0,057$ & & $(1,08)$ & $-0,044$ & & $(0,85)$ & $-0,059$ & & $(1,12)$ \\
\hline \multicolumn{10}{|l|}{ Schulkontext } \\
\hline Gesamtschule & 0,284 & * & $(2,20)$ & & & & 0,577 & & $(1,26)$ \\
\hline Gymnasium & 0,052 & & $(0,45)$ & & & & $-0,367$ & & $(0,81)$ \\
\hline \multicolumn{10}{|l|}{ Ressourcen im Schulkontext } \\
\hline Gesamtschule * Schulerfahrung & & & & & & & $-0,277$ & & $(1,05)$ \\
\hline Gesamtschule * Beziehungen zu Erwachsenen & & & & & & & $-0,055$ & & $(0,20)$ \\
\hline Gesamtschule * Beziehungen zu Gleichaltrigen & & & & & & & $-0,104$ & & $(0,23)$ \\
\hline Gesamtschule * organisierte Aktivitäten & & & & & & & $-0,080$ & & $(0,30)$ \\
\hline Gymnasium * Schulerfahrung & & & & & & & 0,326 & & $(1,35)$ \\
\hline Gymnasium * Beziehungen zu Erwachsenen & & & & & & & $-0,146$ & & $(0,58)$ \\
\hline Gymnasium * Beziehungen zu Gleichaltrigen & & & & & & & $-0,059$ & & $(0,14)$ \\
\hline Gymnasium * organisierte Aktivitäten & & & & & & & 0,375 & & $(1,31)$ \\
\hline \multicolumn{10}{|l|}{ Kontrollvariablen } \\
\hline Geschlecht (1=weiblich) & $-0,510$ & $* * *$ & $(5,17)$ & $-0,476$ & $* * *$ & $(4,88)$ & $-0,516$ & $* * *$ & $(5,22)$ \\
\hline Alter & $-0,117$ & $* *$ & $(2,91)$ & $-0,100$ & * & $(2,53)$ & $-0,117$ & ** & $(2,88)$ \\
\hline Migrationshintergrund ( $1=$ vorhanden $)$ & 0,120 & & $(1,17)$ & 0,147 & & $(1,45)$ & 0,143 & & $(1,39)$ \\
\hline \multicolumn{10}{|l|}{ Übergänge im Wohlbefinden } \\
\hline Übergang von niedrig zu mittel & $-0,181$ & & $(0,29)$ & 0,190 & & $(0,31)$ & $-0,188$ & & $(0,28)$ \\
\hline Übergang von mittel zu hoch & 1,594 & ** & $(2,51)$ & 1,936 & ** & $(3,19)$ & 1,594 & ** & $(2,38)$ \\
\hline \multicolumn{10}{|l|}{ Modellstatistiken } \\
\hline Fallzahl & 1713 & & & 1713 & & & 1713 & & \\
\hline McFadden Pseudo- $R^{2}$ & 0,098 & & & 0,087 & & & 0,101 & & \\
\hline $\mathrm{BIC}$ & 3187,0 & & & 3196,8 & & & 3237,6 & & \\
\hline
\end{tabular}

Alle Modelle sind Ordered-Logit-Regressionen für niedriges (Wert 0), mittleres (1) und hohes (2) Wohlbefinden. Berichtet werden Koeffizienten und in Klammern z-Statistiken. ${ }^{* * *} \alpha \leq 0,001 ;{ }^{* *} \alpha \leq 0,01 ;{ }^{*} \alpha \leq 0,05$ 


\subsection{Wohnumgebung als sozialräumlicher Kontext des Wohlbefindens}

Der dritte Kontext des Wohlbefindens von Kindern und Jugendlichen ist die Wohnumgebung. Als räumlicher Kontext besteht hierbei die Schwierigkeit der Abgrenzung. Theoretisch ist damit der Raum gemeint, in dem sich Kinder aufhalten und ihre außerschulische Freizeit verbringen. Dieser Raum ist jedoch individuell für jedes Kind anders zugeschnitten. Methoden zur Erhebung dieser individuellen Räume wie subjektive Landkarten und die Nadelmethode ${ }^{11}$ sind allerdings für einen forschungsökonomisch sinnvollen Einsatz im UWE-Instrument wenig geeignet. Darüber hinaus sind sie für die Sozialraumplanung schwierig zu integrieren. Deshalb werden städtische Teilgebiete definiert und den darin lebenden Kindern und Jugendlichen als deren Wohnumgebung zugewiesen. In Herne greifen wir auf 32 statistische Bezirke als Wohnumgebungen der Kinder zurück. Dies sind administrativ festgelegte städtische Gebiete, die sich in ihrer Bevölkerungszusammensetzung und Sozialstruktur unterscheiden. Die Sozialstruktur der Bevölkerung eines statistischen Bezirks wird abgebildet durch die demographische Zusammensetzung, gemessen durch den Anteil der unter 18-Jährigen, die ethnische Zusammensetzung, gemessen am Anteil der Personen mit Migrationshintergrund, die soziale Zusammensetzung, gemessen an den Anteilen der Arbeitslosen und der Bedarfsgemeinschaften, sowie die residentielle Stabilität, gemessen an den Anteilen des jährlichen Zuzugs und Wegzugs. Da all diese Merkmale stark miteinander korrelieren, haben wir den Index der sozialen Zusammensetzung gebildet, der den Grad der Deprivation im statistischen Bezirk angibt. Alle Daten basieren auf der aktuell zur Verfügung stehenden Kommunalstatistik und beziehen sich auf den 31. Dezember 2016. Neben den Bestandsdaten wurden auch Daten zur Entwicklung der statistischen Bezirke berücksichtigt, indem die Entwicklung in den letzten fünf Jahren, also zwischen 2011 und 2016, abgebildet wurde. Hierfür wurden die demographische Entwicklung, gemessen über die Veränderung des Anteils der unter 18-Jährigen, die ethnische Entwicklung, gemessen über die Veränderung des Anteils der Personen mit Migrationshintergrund, und die soziale Entwicklung, gemessen über die Veränderung des Anteils der

11 Die Nadelmethode ist ein Verfahren zur Visualisierung von Orten (z. B. Aufenthaltsorte, informelle Treffpunkte, Aneignungsund Streifräume). Kinder und Jugendliche markieren auf Karten von Stadtteilen bzw. Sozialräumen diese Orte mithilfe farbiger Stecknadeln.
Bedarfsgemeinschaften, ermittelt. Die Basis war jeweils das Jahr 2011; Veränderungen wurden in Prozentpunkten gemessen und können theoretisch zwischen -100 und +100 schwanken. Diese Veränderungsdaten korrelieren hochgradig miteinander, sodass diese in einem Index der sozialen Entwicklung zusammengefasst wurden, der den Grad der Veränderung der sozialen Zusammensetzung beschreibt, wobei positive Werte die Entwicklung zu einem deprivierten statistischen Bezirk implizieren.

Wie in den Modellen 1 und 2 der Tabelle $4 \mathrm{zu}$ erkennen ist, sind beide Indices nicht statistisch signifikant und haben somit auch keine Relevanz für das Wohlbefinden. Zugleich bleiben die bisher gefundenen Zusammenhänge der Ressourcen, des Familien- und des Schulkontexts sowie der Kontrollvariablen bestehen, was deren Stabilität und Robustheit verdeutlicht. In Modell 3 der Tabelle 4 sind zusätzlich Interaktionsterme der beiden Indices mit den drei wohnumgebungsspezifischen Ressourcen (Beziehungen zu Erwachsenen, Beziehungen zu Gleichaltrigen und organisierte Aktivitäten) aufgenommen. Keiner dieser Koeffizienten ist signifikant von Null verschieden. Zudem weisen die beiden Ressourcen mit sozialen Beziehungen keine direkten signifikanten Zusammenhänge mit dem Wohlbefinden aus. Das bedeutet, dass die Beziehungen zu Erwachsenen und zu Gleichaltrigen zum Teil über die statistischen Bezirke und damit über die Wohnumgebungen vermittelt mit dem Wohlbefinden zusammenhängen, aber diese Zusammenhänge marginal und damit irrelevant sind.

Abschließend wurden statt der Sozialraumtypen direkt die 32 statistischen Bezirke Hernes berücksichtigt. Dafür wurden wiederum Mehrebenen-Regressionen berechnet, mit Kindern auf der ersten, unteren Ebene und den Bezirken auf der zweiten, oberen Ebene. Dabei zeigte sich, dass es praktisch keine Variationen des Wohlbefindens zwischen den statistischen Bezirken gibt. Das Wohlbefinden ist demnach unabhängig von dem konkret bewohnten statistischen Bezirk.

\section{Zusammenfassung und Ausblick}

Die Ergebnisse aus der Pilotstudie „Wie erleben Kinder sozialräumliche Segregation und Bildungssegregation?" in Herne dienen der Überprüfung der aufgestellten Hypothesen. Hypothese 1 geht davon aus, dass die Wahrscheinlichkeit für ein positives Wohlbefinden umso größer ist, je mehr Ressourcen den Kindern und Jugendlichen zur Verfügung stehen. Sie kann für vier 
Tabelle 4: Regressionen des Wohlbefindens auf stärkende Ressourcen im statistischen Bezirkskontext

\begin{tabular}{|c|c|c|c|c|c|c|c|c|c|}
\hline Variablen & Modell 1 & & & Modell 2 & & & Modell 3 & & \\
\hline \multicolumn{10}{|l|}{ Ressourcen } \\
\hline Ernährung und Schlaf & 0,861 & $* * *$ & $(8,24)$ & & & & 0,862 & *** & $(8,22)$ \\
\hline Schulerfahrungen & 0,806 & $* * *$ & $(7,66)$ & & & & 0,802 & $* * *$ & $(7,61)$ \\
\hline Beziehungen zu Erwachsenen & 0,471 & $* * *$ & $(4,26)$ & & & & 0,044 & & $(0,10)$ \\
\hline Beziehungen zu Gleichaltrigen & 0,787 & $* * *$ & $(4,29)$ & & & & 1,457 & & $(1,95)$ \\
\hline organisierte Aktivitäten & 0,021 & & $(0,18)$ & & & & 0,215 & & $(0,45)$ \\
\hline Summenindex & & & & 0,610 & *** & $(13,39)$ & & & \\
\hline \multicolumn{10}{|l|}{ Familienkontext } \\
\hline Alleinerziehenden-Haushalt & $-0,551$ & *** & $(4,02)$ & $-0,530$ & $* * *$ & $(3,90)$ & $-0,559$ & $* * *$ & $(4,06)$ \\
\hline Anzahl Geschwister & $-0,057$ & & $(1,05)$ & $-0,044$ & & $(0,82)$ & $-0,061$ & & $(1,13)$ \\
\hline \multicolumn{10}{|l|}{ Schulkontext } \\
\hline Gesamtschule & 0,278 & * & $(2,10)$ & 0,294 & * & $(2,24)$ & 0,280 & * & $(2,11)$ \\
\hline Gymnasium & 0,047 & & $(0,40)$ & $-0,009$ & & $(0,08)$ & 0,042 & & $(0,35)$ \\
\hline \multicolumn{10}{|l|}{ Kontext statistischer Bezirke } \\
\hline Index sozialer Zusammensetzung 2016 (ISZ) & 0,005 & & $(0,34)$ & 0,013 & & $(0,86)$ & 0,048 & & $(0,83)$ \\
\hline Index sozialer Entwicklung 2011-2016 (ISE) & 0,010 & & $(0,96)$ & 0,007 & & $(0,65)$ & 0,013 & & $(0,33)$ \\
\hline \multicolumn{10}{|l|}{ Ressourcen im Kontext statistischer Bezirke } \\
\hline ISZ * Beziehungen zu Erwachsenen & & & & & & & 0,037 & & $(1,14)$ \\
\hline ISZ * Beziehungen zu Gleichaltrigen & & & & & & & $-0,057$ & & $(1,04)$ \\
\hline ISZ * organisierte Aktivitäten & & & & & & & $-0,000$ & & $(0,00)$ \\
\hline ISE * Beziehungen zu Erwachsenen & & & & & & & $-0,011$ & & $(0,44)$ \\
\hline ISE * Beziehungen zu Gleichaltrigen & & & & & & & 0,017 & & $(0,44)$ \\
\hline ISE * organisierte Aktivitäten & & & & & & & $-0,023$ & & $(0,99)$ \\
\hline \multicolumn{10}{|l|}{ Kontrollvariablen } \\
\hline Geschlecht (1=weiblich) & $-0,513$ & $* * *$ & $(5,10)$ & $-0,472$ & $* * *$ & $(4,74)$ & $-0,506$ & $* * *$ & $(5,02)$ \\
\hline Alter & $-0,113$ & ** & $(2,75)$ & $-0,097$ & * & $(2,42)$ & $-0,114$ & ** & $(2,76)$ \\
\hline Migrationshintergrund (1=vorhanden) & 0,090 & & $(0,83)$ & 0,104 & & $(0,97)$ & 0,097 & & $(0,90)$ \\
\hline \multicolumn{10}{|l|}{ Übergänge im Wohlbefinden } \\
\hline Übergang von niedrig zu mittel & 0,004 & & $(0,01)$ & 0,408 & & $(0,64)$ & 0,608 & & $(0,61)$ \\
\hline Übergang von mittel zu hoch & 1,790 & ** & $(2,70)$ & 2,168 & *** & $(3,40)$ & 2,397 & * & $(2,38)$ \\
\hline \multicolumn{10}{|l|}{ Modellstatistiken } \\
\hline Fallzahl & 1647 & & & 1647 & & & 1647 & & \\
\hline McFadden Pseudo- $R^{2}$ & 0,095 & & & 0,084 & & & 0,096 & & \\
\hline $\mathrm{BIC}$ & 3097,2 & & & 3102,8 & & & 3138,1 & & \\
\hline
\end{tabular}

Alle Modelle sind Ordered-Logit-Regressionen für niedriges (Wert 0), mittleres (1) und hohes (2) Wohlbefinden. Berichtet werden Koeffizienten und in Klammern z-Statistiken. ${ }^{* * *} \alpha \leq 0,001$; ${ }^{* *} \alpha \leq 0,01 ;{ }^{*} \alpha \leq 0,05$ 
der fünf Ressourcen (Ernährung und Schlaf, Schulerfahrungen, Beziehungen zu Erwachsenen und Beziehungen zu Gleichaltrigen) bestätigt werden und trifft für den Summenindex vollständig zu. Lediglich für organisierte Freizeitaktivitäten gilt dies nicht. Hypothese 2 beschreibt einen geringen Unterschied zwischen Alleinerziehenden-Haushalten und Paarhaushalten in Bezug auf den Zusammenhang von Ressourcenausstattung und Wohlbefinden. Die Ergebnisse beschreiben auch, wie wichtig der Familienkontext ist. Dies gilt jedoch nur für die Differenzierung nach Alleinerziehenden-Haushalten. Es zeigt sich, dass Kinder aus Alleinerziehenden-Haushalten ein substanziell geringeres Wohlbefinden aufweisen als Kinder aus anderen Haushalten. Allerdings besteht kein Zusammenhang zwischen dem Wohlbefinden und der Anzahl der Geschwister. Hypothese 3, nach der das Wohlbefinden schulspezifische Unterschiede aufweist, kann in Form der Schultypen bestätigt werden. So gibt es keinen substanziellen Unterschied im Wohlbefinden der Kinder aus Haupt- und Realschulen einerseits und Gymnasien andererseits. Dagegen haben Kinder, die Gesamtschulen besuchen, ein signifikant höheres Wohlbefinden als Kinder von Haupt- und Realschulen. Dass die Wohnumgebung einen Einfluss auf das Wohlbefinden hat (Hypothese 4), wurde widerlegt, da es keine systematischen Unterschiede zwischen den statistischen Bezirken gibt. Dies kann daran liegen, dass Herne als eine arme Stadt zu klassifizieren ist, in der viele der 32 statistischen Bezirke von Armut geprägt sind und somit die soziale Segregation gering ausgeprägt ist. Ein weiteres Problem kann in der falschen Operationalisierung der Wohnumgebung liegen. Es bleibt kritisch zu hinterfragen, ob die Wohnumgebung mit der Zugehörigkeit zum statistischen Bezirk einhergeht. Möglicherweise ist eine Operationalisierung als individuell erlebte Wohnumgebung zielführender. Damit schafft man allerdings ein Folgeproblem, denn individuelle Wohnumgebungen sind nicht als stadtentwicklungsrelevante Sozialräume darstellbar. Für die kommunalpolitische Umsetzung dieser Ergebnisse wäre somit keine Ausrichtung an Sozialräumen, sondern eher an Familien und, abgeschwächt, an Schulen angezeigt.

Die Pilotstudie hat zahlreiche Erkenntnisse über die Zustände und Zusammenhänge des kindlichen Wohlbefindens in einer deutschen Stadt aufgezeigt. Um das Instrument jedoch als kommunales Monitoring einer integrierenden Stadtentwicklung zu etablieren, sind weitere Schritte notwendig. Dazu gehört, die wissenschaftlichen Erkenntnisse an die relevanten kommunalen, schulischen und zivilgesellschaftlichen Akteure zu transferieren, um Interventionen und Veränderungen anzuregen sowie Präventionsmaßnahmen einzuleiten. Um diese Prozesse in Gang zu setzen, werden zum einen die Daten durch das „Zentrum für interdisziplinäre Regionalforschung" (ZEFIR) in den Schulen vorgestellt und zum anderen vom Bildungsbüro Herne Schulworkshops angeboten, die von den Herner Schulen in Anspruch genommen werden können. In diesen setzen sich die Kinder und Jugendlichen, aber auch Lehrer aktiv mit den Ergebnissen auseinander und leiten Interventionsmaßnahmen ab. Zudem werden die Ergebnisse in einer stadtübergreifenden Schulpflegschaft vorgestellt und auch in den kommunalen Ausschüssen für Schule sowie Kinder, Jugendliche und Familie präsentiert. So soll die Weiterarbeit mit unterschiedlichen Akteuren aus verschiedenen Bereichen angeregt werden.

Schließlich bleibt festzuhalten, dass UWE zukünftig in weiteren Kommunen eingesetzt und in Herne als Evaluationsinstrument fortgeführt werden soll. Das Ziel ist es, ein durch Kommunen finanzierbares und handhabbares Monitoring-Instrument zu entwickeln. Dies wird nicht über eine sehr zeit- und kostenintensive Datenerhebung wie in der Pilotstudie gelingen. Deshalb wird in einer nächsten Stufe UWE deutlich effizienter gestaltet, indem zum Beispiel eine Online-Befragung, routinierte Auswertungen und standardisierte Berichtslegungen implementiert werden.

\section{Funding Information}

Dieser Beitrag ist im Rahmen des Forschungsprojekts „Wie erleben Kinder sozialräumliche Segregation und Bildungssegregation?" entstanden. Es wurde durch das Forschungsinstitut für gesellschaftliche Weiterentwicklung (FGW) des Landes Nordrhein-Westfalen gefördert (Förderkennzeichen 005-1605-0020) und vom Zentrum für interdisziplinäre Regionalforschung (ZEFIR) der Ruhr-Universität Bochum durchgeführt.

\section{Literatur}

Beisenkamp, A.; Klöckner, C.; Hallmann, S.; Preißner, C. (2009): LBS-Kinderbarometer Deutschland 2009. Stimmungen, Trends und Meinungen von Kindern aus Deutschland. Recklinghausen.

Bertram, H.; Stein, T.; Niejahr, E. (2011): Starke Kinder - starke Familien. Wohlbefinden von Kindern in Städten und Gemeinden. Studie im Auftrag der Robert-Bosch-Stiftung. Stuttgart..

BMFSFJ - Bundesministerium für Familie, Senioren, Frauen und Jugend (2012): Alleinerziehende in Deutschland - Lebenssi- 
tuationen und Lebenswirklichkeiten von Müttern und Kindern. Berlin. = Monitor Familienforschung 28.

BMFSFJ - Bundesministerium für Familie, Senioren, Frauen und Jugend (2015): Dossier Wohlergehen von Familien. Berlin.

Bronfenbrenner, U. (1972): Two Worlds of Childhood. U.S. and U.S.S.R. Harmondsworth.

Bronfenbrenner, U. (1981): Die Ökologie der menschlichen Entwicklung: Natürliche und geplante Experimente. Stuttgart.

Dalbert, C.; Stöber, J. (2005): Belief in a just world and distress at school. In: Social Psychology of Education 8, 2, 123-135. doi: 10.1007/s11218-005-1835-2

Eder, F. (2004): Der Einfluss einzelner Lehrpersonen auf das Befinden von Schülerinnen und Schülern. In: Hascher, T. (Hrsg.): Schule positiv erleben. Ergebnisse und Erkenntnisse zum Wohlbefinden von Schülerinnen und Schülern. Bern, 91-112.

Esser, H. (2001): Integration und ethnische Schichtung. Mannheim. = Arbeitspapiere - Mannheimer Zentrum für Europäische Sozialforschung 40 .

Groos, T. (2015): Gleich und gleich gesellt sich gern. Zu den sozialen Folgen freier Grundschulwahl. Gütersloh. = Schriftenreihe Arbeitspapiere wissenschaftliche Begleitforschung „Kein Kind zurücklassen!" 5.

Groos, T.; Jehles, N. (2015): Der Einfluss von Armut auf die Entwicklung von Kindern. Ergebnisse der Schuleingangsuntersuchung. Gütersloh. = Schriftenreihe Arbeitspapiere wissenschaftliche Begleitforschung „Kein Kind zurücklassen!“ 3.

Hallmann, S.; Beisenkamp, A.; Klöckner, C.; Preißen, C. (2008): Kinderumfrage Frankfurt a.M. Stimmungen, Meinungen, Trends von Kindern in Frankfurt. Ergebnis der Erhebung 2008. Herten.

Hascher, T. (2004): Wohlbefinden in der Schule. Münster. = Pädagogische Psychologie und Entwicklungspsychologie 40.

Hilke, M. (2013): Wie arme Kinder die Unterstadt erleben. Ungleiche Kindheiten und städtischer Raum in Oberhausen und Windhoek. Bochum. = ZEFIR-Forschungsberichte 4.

Hübner, N.; Wagner, W.; Kramer, J.; Nagengast, B.; Trautwein, U. (2017): Die G8-Reform in Baden-Württemberg: Kompetenzen, Wohlbefinden und Freizeitverhalten vor und nach der Reform. In: Zeitschrift für Erziehungswissenschaft 20, 4, 748-771. doi: 10.1007/s11618-017-0737-3

Kaufmann, F.-X.; Herlth, A.; Strohmeier, K. P. (1980): Sozialpolitik und familiale Sozialisation. Zur Wirkungsweise öffentlicher Sozialleistungen. Stuttgart. $=$ Schriftenreihe des Bundesministers für Jugend, Familie und Gesundheit 76.

Kershaw, P.; Irwin, L.; Trafford, K.; Hertzman, C. (2005): The British Columbia Atlas of Child Development. Georgetown.

Kothe, M. (2014): Kinder als endogene Potenziale bei Quartiersentwicklung. Wie Kinder in benachteiligten Stadtteilen ihre Quartiere sehen und was der Blick für die Quartiersentwicklung bedeutet. Abschlussarbeit zur Erlangung des Master of Arts im Studiengang Real Estate/Business Administration an der EBZ Business School, University of Applied Sciences, Bochum.

Kullmann, H.; Geist, S.; Lütje-Klose, B. (2015): Erfassung schulischen Wohlbefindens in inklusiven Schulen. In: Kuhl, P.; Stanat, P.; Lütje-Klose, B.; Gresch, C.; Pant, H. A.; Prenzel, M. (Hrsg.): Inklusion von Schülerinnen und Schülern mit sonderpädagogischem Förderbedarf in Schulleistungserhebungen. Wiesbaden, 301-333. doi: 10.1007/978-3-658-06604-8
Meister-Wolf, M. (2003): Vergleich von Ganztagsvolksschule und ,Halbtagsschule' in Bezug auf das Wohlbefinden der Schülerlnnen. München.

OECD - Organisation for Economic Co-operation and Development (2018): PISA Sonderauswertung Resilienz. http://www. oecd.org/berlin/publikationen/VSD_OECD_Erfolgsfaktor\%20 Resilienz.pdf (05.03.2019).

Schonert-Reichl, K. A.; Guhn, M.; Gadermann, A. M.; Hymel, S.; Sweiss, L.; Hertzman, C. (2013): Development and Validation of the Middle Years Development Instrument (MDI): Assessing Children's Well-Being and Assets across Multiple Contexts. In: Social Indicators Research 114, 2, 345-369. doi: 10.1007/ s11205-012-0149-y

Strohmeier, K. P. (2010): Durchschnitt ist nirgends - Segregation und die Gesundheit von Kindern in der Stadt. In: Dahme, H.-J.; Wohlfahrt, N. (Hrsg.): Systemanalyse als politische Reformstrategie. Wiesbaden, 318-334. doi: 10.1007/978-3-53192013-9

Strohmeier, K. P.; Gehne, D. H.; Bogumil, J.; Micosatt, G.; von Görtz, R. (2016): Die Wirkungsweise kommunaler Prävention: Zusammenfassender Ergebnisbericht der wissenschaftlichen Begleitforschung. Gütersloh.

Strohmeier, K. P.; Gehne, D. H.; Groos, T.; Jehles, N. (2014): Die fachliche Begleitforschung. Konzept und erste Ergebnisse. Gütersloh. = Schriftenreihe Arbeitspapiere wissenschaftliche Begleitforschung „Kein Kind zurücklassen!“ 2.

Stubbe, T. C.; Bos, W.; Euen, B. (2012): Der Übergang von der Primar- in die Sekundarstufe. In: Bos, W.; Tarelli, I.; BremerichVos, A.; Schwippert, K. (Hrsg.): IGLU 2011. Lesekompetenzen von Grundschulkindern in Deutschland im internationalen Vergleich. Münster, 209-226.

Terpoorten, T. (2014): Räumliche Konfiguration der Bildungschancen. Segregation und Bildungsdisparitäten am Übergang in die weiterführenden Schulen im Agglomerationsraum Ruhrgebiet. Bochum. = ZEFIR-Materialien 3.

Thomson, K. C.; Oberle, E.; Gadermann, A. M.; Guhn, M.; Rowcliffe, P.; Schonert-Reichl, K. A. (2017): Measuring socialemotional development in middle childhood: The Middle Years Development Instrument. In: Journal of Applied Developmental Psychology 55, 107-118. doi: 10.1016/j.appdev.2017.03.005

Walper, S.; Wendt, E.-V. (2005): Nicht mit beiden Eltern aufwachsen - ein Risiko? In: Alt, C. (Hrsg.): Kinderleben - Aufwachsen zwischen Familie, Freunden und Institutionen. Band 1: Aufwachsen in Familien. Wiesbaden, 187-216.

Wößmann, L. (2009): International Evidence on School Tracking: A Review. In: CESifo DICE Report 7, 1, 26-34.

ZEFIR - Zentrum für interdisziplinäre Ruhrgebietsforschung (2008): Familienbericht Herne 2007. Lebenslage und Zufriedenheit von Familien. Bochum. 